\title{
An Energy-Efficient Routing Method of Wireless Sensor Networks
}

\author{
Sun Ting ${ }^{1}$, Lu Dongxin ${ }^{2}$, and Yang Yongtian ${ }^{1}$ \\ ${ }^{1}$ College of Computer Science and Technology, Harbin Engineering University, Harbin \\ 150001, China \\ \{sunting, yangyongtian\} @hrbeu.edu.cn \\ ${ }^{2}$ ZTE Software Engineering, The Torch Street 999, Nan Chang 330029, China \\ lu.dongxinaztenc.com.cn
}

\begin{abstract}
In wireless sensor networks(WSNs), as sensor nodes are characterized by having specific requirements such as limited energy availability, low memory and reduced processing power, energy efficiency is a key issue in designing the network routing. In the existing clustering-based routing protocols for the WSNs, the cluster-heads are usually selected randomly and do a lot of work, which may cause unbalanced energy consumption and thus short network lifetime. Based on this approach, the paper proposes a method that a clusterhead distributes energy load evenly among its members based on their energy usage by chain. With the chain, in a cluster, different node is selected as Agent Node to send the data to cluster-head at different cluster cycle. The new method balances the nodes' power depletion. Simulation results show that it has better power management performance and can prolong the lifetime of the network.
\end{abstract}

Keywords: Wireless Sensor Networks, Routing Method, Cluster, Chain.

\section{Introduction}

As a distributed sensing network, wireless sensor networks consist of many small intelligent types of equipment [1]. Since sensor nodes carry limited and generally irreplaceable power source, the routing protocols designed for the WSN must take the issue of energy saving into consideration. Clustering-based routing protocol is a popular protocol proposed for the WSN to minimize the consumption of the energy of the sensors.

There have existed many cluster-based routing protocols [2][3], but they still have many problems. In this paper, based on the cluster-based routing model, we propose a new routing transmission method, with which a cluster-head distributes energy load evenly among its members based on their energy usage by chain.

This paper is organized as follows. Related works are introduced in Section 2. In Section 3, we present the new routing method and its building process. Simulation results are given in Section 4. Finally we summarize the main results and discuss future research direction in Section 5. 


\section{Related Works}

Low Energy Adaptive Clustering Hierarchy (LEACH), described in [2], is probably one of the more referenced protocols in the sensor networks area. The recent researches on the routing with the hierarchical structure such as PEACH [4] and EDACH [5] employ a similar approach as LEACH. As an improved version to LEACH, LEACH-C [6] uses a centralized clustering algorithm to produce better clusters, thus achieves better performance.

The transmission model used in LEACH can lighten the centralized consumption of nodes' energy to a certain extent. However, the randomly election of the cluster-heads can cause unbalance energy consumption in the nodes. On the other hand, in the cluster, the nodes near the cluster-head still need to transfer other nodes' data just like the routers and need more energy. Sub-clustering method [3] improves the LEACH partly, but increases the computation work and management load of the cluster-head. In order to resolve these problems, in this paper we present a new routing method with which a chain is built in every cluster, which can resolve these problems better.

\section{Chain in Cluster}

Each nodes i maintains a unique identification, ID(i), a cluster identification to which i belongs, CID(i), and its remaining battery power, CRP(i). Its location is L(i). After the clusters are built, the cluster-head check all the member nodes' information and select the node with most residual energy as its' agent node. At the same time the cluster-head broadcasts all nodes location information in the cluster, every node select the closest neighbor to form a chain according to these information. When the clusterhead receives the data collection request from the sink, the agent node transfers the request to the two ends of the chain and the data begin to flow from the two ends to it. At the same time, the middle nodes will aggregate these data and finally the agent node transfers these data to cluster-head and cluster-head transfers them to sink node.

As shown in Figure 1, the method balances the nodes' power consumption.

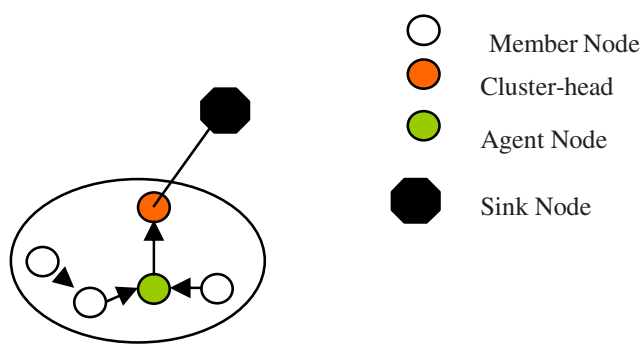

Fig. 1. One Node as Agent Node

As the agent node consumes more energy than other nodes within the chain, the cluster-head elects another node with the most residual power as agent node in next cluster cycle. That is, when a cluster cycle is over, nodes are informed about their newly elected agent node. Nodes within a chain transmit to their newly elected agent node. 


\section{Simulation Results}

For the simulation, we use the OPNET network simulator. The environment consists of 150 nodes distributed randomly in a $100 \mathrm{~m} \times 100 \mathrm{~m}$ area. Each node is equipped with the initial energy of 5 joules. We compare the new method with LEACH routing method and recent research work Sub-cluster routing method.

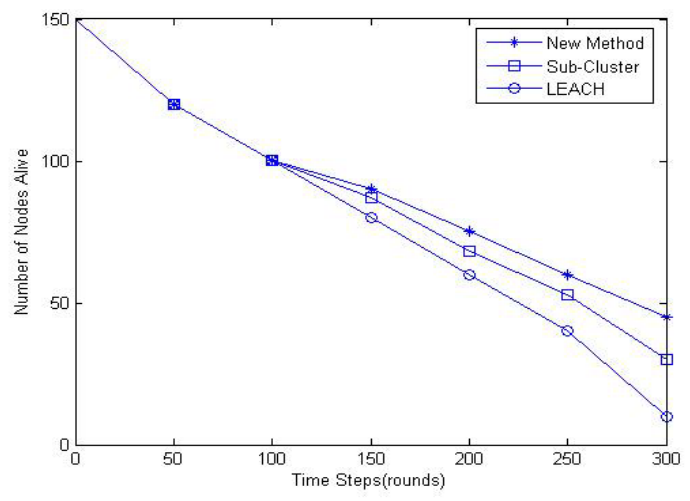

Fig. 2. Number of Nodes Alive

From the Figure 2, we can draw a conclusion that at first new method has the similar performance with other methods. However, with the increase of number of rounds, new approach has better result than conventional approaches. The reason is that, in LEACH, cluster-heads not only transmit the data to sink, but also aggregates all the information that accepted from the member nodes, which need more energy. And in LEACH, the nodes near the cluster-head, as routers to transmit other nodes' data also need more energy. These works can make the nodes die soon. In sub-cluster method, the cluster-head also need to aggregate the information that accepted from the sub-cluster-heads. In our new method, the work is distributed to the member nodes and the data attending cluster-head has been aggregated. With this method, all the nodes in the cluster have more balanced power consumption. The number of nodes alive is larger.

Figure 3 shows us the results of nodes' power consumption with the change of network distribution range. When the distribution range of network is small and the nodes density is high, new model and sub-cluster method have not better performance than traditional model, because they have more computation to form the chain and sub-cluster in the cluster. With the increase of network distribution range, the transmission distance between the nodes increases also. In LEACH, the member nodes of a cluster have to transmit their data to cluster-head with long distance. The sub-cluster method improves it by reducing the overhead of re-clustering and shorter transmission distance. With our method, the nodes of the chain only need forward their data to the nearby neighbor. New method shows us its advantages. It has lower power consumption and will prolong the lifetime of network. 


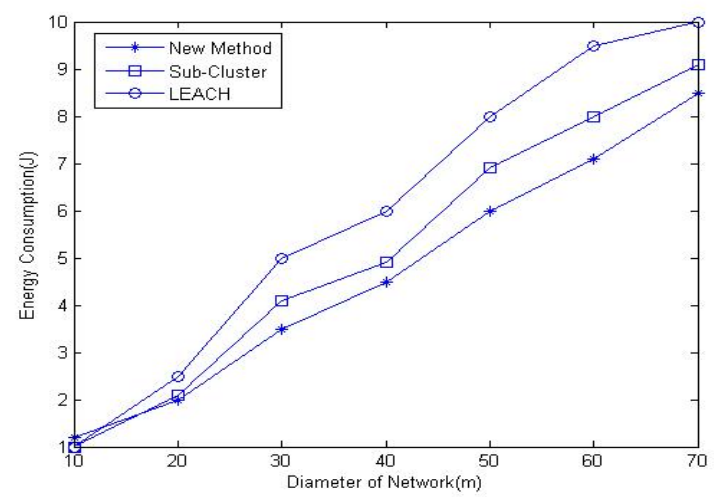

Fig. 3. Energy Consumption

\section{Conclusion and Future Works}

In this paper, we introduced a new method to prolong the lifetime of WSNs. Simulation results show that the new method has better performance when the network range is increased. But as we all know, the routing protocol of WSNs is application-based. We will study the performance of our approach under various conditions. For example, we will consider nodes' mobility and fault. So, we should do more things to improve the performance of the new method in the future.

\section{References}

1. Hill J, Szew Czyk R, Woo A, Hollar S. System architecture directions for networked sensors. Proceedings of the 9th ACM International Conference on Architectural Support for Programming Languages and Operating Systems. 2000, 93-104

2. W. Heinzelman, A. Chandrakasan, Hbalakrishnan. Energy-efficient communication protocol for wireless microsensor networks. The Hawaii Int'l Conf. System Sciences, Hawaii, IEEE Computer Society, 2000

3. Kyung-Won Nam, Jun Hwang, Cheo-Min Park, Young-Chan Kim. Energy-Efficiency Method for Cluster-Based Sensor Networks. ICCSA 2005, LNCS 3480, 2005, pp 11701176

4. K.T. Kim and H.Y. Youn.: PEACH: Proxy-Enable Adaptive Clustering Hierarchy for Wireless Sensor network: Proceeding of The 2005 International Conference On Wireless Network, pp. 52-57, June 2005.

5. K.T. Kim and H. Y. Youn.: Energy-Driven Adaptive Clustering Hierarchy (EDACH) for wireless sensor networks: EUC LNCS3823, pp. 1098-1107, 2005.

6. Heinzelman, W., Chandrakasan, A., Balakrishnan, H.: An Application-Specific Protocol Architecture for Wireless Microsensor Networks. IEEE Trans. on Wireless Communications 1(4) (2002) 660-670 